\title{
Using Syndromic Surveillance to Characterize Unintentional Ingestions in Children
}

\author{
Alyssa Z. Chase*1, Mansi Agarwal' ${ }^{1}$, Maria Mercurio-Zappala ${ }^{2}$ and Mark Su² \\ ${ }^{1}$ Syndromic Surveillance, NYC Department of Health, Long Island City, NY, USA; ${ }^{2}$ NYC Poison Control Center, New York, NY, USA
}

\section{Objective}

To describe unintentional ingestions (UIs) in children $<5$ years using syndromic data from emergency departments in New York City (NYC) from 2010 to 2014.

\section{Introduction}

UIs are among the leading causes of injury in children younger than 5 years in $\mathrm{NYC}^{1}$. About 3000 calls are received each year by the NYC Poison Control Center (PCC) for this age group ${ }^{1}$. Common UI exposures include medications, cosmetics, household cleaners, foreign bodies, and pesticides ${ }^{2}$. We examined UIs in NYC from January 2010 to July 2014 for children $<5$ years to investigate the utility of syndromic surveillance in conjunction with the PCC in capturing real-time pediatric UIs over time.

\section{Methods}

Chief complaint free-text and ICD9 codes from the NYC syndromic surveillance system were scanned for keywords related to UIs, including "ACCIDENTAL INGEST" and "POISONING" in children $<5$ years. Chi-square tests were used to assess weekday vs. weekend, month, and year trends in types of UIs from 2010 to 2013. Neighborhood poverty level (based on patient zip code) was defined as the percent of residents with incomes below $100 \%$ of the Federal Poverty Level (per American Community Survey [ACS] 2008-2012) and grouped into 4 categories: $<10 \%, 10 \%$ to $<20 \%, 20 \%$ to $<30 \%$, or $\geq 30 \%$ in poverty. Using a Cochran-Armitage trend test, we compared the proportion of the population $<5$ years-old (per Census 2010) with a UI visit across neighborhood poverty levels. A multivariable negative binomial regression model was used to examine the association between the zip code-level number of medicinal UIs among $<5$ yearolds with the proportion of all children $<6$ living with a grandparent (per ACS 2008-2012 ), adjusting for neighborhood-level poverty; the offset term was the log of the total population of $<5$ year-olds.

\section{Results}

We identified 11,605 UIs from over 2 million ED visits for children $<5$ years. Mean age was 1.8 years (median 2 years), and $53 \%$ were males. UIs involving foreign bodies, notably coins, were the most common visit type (39\%), followed by visits for medications (20\%). Analgesics were the most commonly mentioned medication, though $48 \%$ of all UI visits for medications did not specify the medication. These trends did not vary significantly by weekday vs. weekend or by month. There were significant annual differences in types of UI visits $(\mathrm{p}<0.0001)$ and in types of medicinal UI visits $(\mathrm{p}=0.01)$. Between 2010 and 2014, UIs of pesticides decreased from $4.6 \%$ to $2.6 \%$ while coin UI increased from $5.4 \%$ to $10.7 \%$. Reporting UIs as "unknown" also increased from $19.6 \%$ to $24.6 \%$. For the medicinal visits, the observed association between year and medicinal type was attenuated after "unknown" and "other" medicine types were removed from the analysis. The proportion of children $<5$ years with UI visits increased with increasing neighborhood poverty level $(\mathrm{p}<0.001)$. We also found a positive association between the rate of medicinal UIs per 1,000 children and the proportion of children $<6$ living with a grandparent in a given zip code $(\mathrm{p}<0.001)$.

\section{Conclusions}

NYC EDs see over 2500 visits for UIs per year among children $<5$ years, similar to the number of calls the PCC receives annually for the same age group. Moreover, the PCC also listed analgesics as the most commonly reported medication-related call, and similarly found an association between lower neighborhood income and higher UI rates ${ }^{1}$. Taken together, our results suggest syndromic data are representative of reported NYC UIs in children. Further work will determine congruence between PCC calls and ED visits, with the ultimate goal of improving the completeness of real-time UI surveillance in NYC.

\section{Keywords}

syndromic surveillance; poisoning; poison control center; pediatrics

\section{References}

1. Wheeler K, Hoffman R, Kass D, Leighton J. Unintentional Poisoning in New York City Children. NYC Vital Signs 2009, 8(2): 1-4.

2. The New York City Department of Health and Mental Hygiene. Household Poisons \& Kids. Accessed 13 August 2014. http://www. nyc.gov/html/doh/html/environmental/poison-home-kids.shtml

3. United States Census Bureau / American FactFinder. "B10001 : Grandchildren Under 18 Years Living with a grandparent Householder by Age of Grandchild" 2008 - 2012 American Community Survey. U.S. Census Bureau's American Community Survey Office, 2012. Web. 25 August $2014<$ http://factfinder2.census.gov〉.

\footnotetext{
*Alyssa Z. Chase

E-mail: achase@health.nyc.gov
} 